\title{
CLASSICAL STOCHASTIC APPROACH TO COSMOLOGY REVISITED
}

\author{
Moncy V John ${ }^{\dagger}$ Sivakumar $\mathrm{C}^{\ddagger}$ and K. Babu Joseph \\ Department of Physics, Cochin University of Science and Technology, Kochi \\ 682022, India
}

Short title : Stochastic Approach To Cosmology

\begin{abstract}
The classical stochastic model of cosmology recently developed by us is reconsidered. In that approach the parameter $w$ defined by the equation of state $p=w \rho$ was taken to be fluctuating with mean zero and we compared the theoretical probability distribution function(PDF) for the Hubble parameter with observational data corresponding to a universe with matter and vacuum energy. Eventhough qualitative agreement between the two was obtained, an attempt is herein made to introduce a more realistic assumption for the mean of $w$ and use it for the calculations. In the present theory the mean values of both $p$ and $w$ are taken to be nonzero. The theoretical and observational PDFs are compared for different epochs and values of the Hubble parameter. The corresponding values of the diffusion constant $D$ obtained are approximately constant. We use the scatter in the observed redshift-magnitude data of Type Ia supernova to place limits on the stochastic variation in expansion rate and consequently, on the stochastic variation of the equation of state.
\end{abstract}

PACS numbers: 98.65 Dx, 98.80 Es, 02.50 Ey

† Permanent Address: Department of Physics, St. Thomas College, Kozhencherri - 689641, Kerala, India. Email : moncy@stthom.ernet.in

‡ e-mail: sivakumarc@cusat.ac.in 


\section{Introduction}

The uncertainty in the determination of the Hubble parameter $\mathrm{H}$, which is a measure of the expansion rate of the universe, is one of the most intriguing issues in the history of cosmology. The origin of the uncertainty is obvious from the redshift-magnitude diagram (Hubble diagram); despite rigorous attempts to control the random errors in measurement, there is a clear scatter in it, though it is now possible to narrow down this to a great extent. But, as we show later in this paper by using the redshift-apparent magnitude data for Type Ia supernovae (SNe Ia) [1], the scatter increases as we go to higher redshifts. In a recent work [2], we have attempted to explain this scatter as arising from an inherent stochastic or nondeterministic nature of the Hubble parameter. It was shown that a fluctuating $w$-factor in the equation of state $p=w \rho$ will lead to this kind of behaviour for $H$. In order to arrive at the notion of a stochastic equation of state, we consider the following. Several recent measurements $[1,3,4,5,6]$ indicate that the present universe contains components other than ordinary matter and radiation, like vacuum energy, quintessence etc.. Let the equation of state for each component, with density $\rho_{i}$, be written as $p_{i}=w_{i} \rho_{i},(i=1,2, .$.$) , where p_{i}$ is the corresponding pressure $\left(w_{i}=0\right.$ for dust, $1 / 3$ for radiation, -1 for vacuum energy, etc.; in

general, $\left.-1<w_{i}<+1\right)$. The total energy density is $\rho=\sum_{i} \rho_{i}$ and the total pressure $p=\sum_{i} p_{i}$. From the law of conservation of total energy-momentum 
tensor expressed in the form

$$
\begin{aligned}
\dot{\rho}=\sum_{i} \dot{\rho}_{i}=-3 \frac{\dot{a}}{a}(\rho+p) & =-3 \frac{\dot{a}}{a} \rho(1+w) \\
& =-3 \frac{\dot{a}}{a} \sum_{i} \rho_{i}\left(1+w_{i}\right)
\end{aligned}
$$

which follows from the Einstein equation and one can write the total $w$-factor as

$$
w \equiv \frac{p}{\rho}=-\frac{\sum_{i} \dot{\rho}_{i}}{3(\dot{a} / a) \rho}-1=\sum_{i} \frac{\rho_{i}}{\rho}\left(1+w_{i}\right)-1,
$$

where $a$ is the scale factor. The conservation of individual components, which may be expressed as $\dot{\rho}_{i}=-3(\dot{a} / a) \rho_{i}\left(1+w_{i}\right)$, is only an extra assumption since it does not follow from the Einstein's field equation. Equivalently, it can be stated that in a many component fluid as in the above case, the Einstein equations, along with the equations of state of individual components are insufficient to determine the individual $\dot{\rho}_{i}$ 's. Thus it is more general not to assume conservation of individual components and this will lead to creation of one component at the expense of other components. Since they are not uniquely determined by the field equations, such creation can be considered as sporadic events, like those occurring in galactic nuclei, which can result in fluctuations in the ratio $\rho_{i} / \rho$. Here we make the assumption that this will lead to a stochastic equation of state, as seen from equation (2) above. Consequently, also the expansion rate will be fluctuating and the equation for the Hubble parameter will appear as a Langevin type [7] equation. Recently Padmanabhan [8] has proposed a similar scenario in which the observed cos- 
mological constant arises from quantum fluctuations of some energy density; consequently the FRW equations are to be solved with a stochastic term on the right hand side. In [2], it was assumed for the sake of simplicity that space sections are flat and $w$ is a Gaussian $\delta$-correlated stochastic force with zero mean. With these assumptions, we have written the Fokker-Planck (FP) equation, whose solution gives the theoretical probability distribution function (PDF) for $H_{0}$ at time $t_{0}$, denoted as $W\left(h, t_{0}\right)$ (where $H_{0}=100 h \mathrm{~km}$ $\mathrm{s}^{-1} \mathrm{Mpc}^{-1}$; the subscript 0 denotes the present epoch). Using the redshiftapparent magnitude data $\mu_{o}$ for SN Ia used in [1], we computed the observational PDF $p\left(h \mid \mu_{o}\right)$ for $h$ in the present universe, again assuming its space sections to be flat. This PDF arises from the point-to-point variance of the Hubble flow. We compared the two plots for the present universe and found them to agree well, for a value of the diffusion constant $D$ appearing in the FP equation equal to $4 \times 10^{13} \mathrm{~s}$.

This result is a first step towards an understanding of the anomalous scatter in the Hubble diagram at high redshifts. However, there are certain refinements to be made in our analysis. One drawback of the above scheme of comparing these two PDF's is that when we derived $W(h, t)$, the assumption was made that $w$ has mean value zero, whereas the observational PDF $p\left(h \mid \mu_{o}\right)$ was evaluated for a model which contains dust and vacuum energy, which has the mean total pressure negative. Instead, if we had used in this evaluation the expression for the distance modulus for a flat universe which is dust 
dominated (i.e., with $w=0$ ), an observational PDF will be obtained, but the best fit value for $h$ would be ridiculously low. (This is all the fuss about the new observations - that they are incompatible with an $\Omega_{\Lambda}=0$ flat model.)

Another shortcoming is that though in both cases we take the PDF for $h$, it remains to be explained how legitimate is the comparison of $W\left(h, t_{0}\right)$ for the present universe with a PDF $p\left(h \mid \mu_{0}\right)$ evaluated using the data that include high redshift objects, which belong to the distant past.

In this paper, we attempt to rectify these two defects and to make a more rigorous test of the stochastic assumptions using observational data by (1) comparing both the theoretical and observational PDF's evaluated for the same model, which is an alternative flat model [9] and (2) evaluating the observational PDF $p\left(h_{j} \mid \mu_{o j}\right)$ for the Hubble parameter at the same epoch $t_{j}$ as that in the theoretical $\mathrm{PDF} W\left(h_{j}, t_{j}\right)$. This procedure helps us to compare the theoretical and observational PDF's for the Hubble parameter for the same model, and at the same epoch. The value of the diffusion constant evaluated at any time is obtained as nearly a constant, in agreement with our assumptions. A novel feature in our present approach is that we evaluate the observational PDF for Hubble parameter at various instants in the past, also with an objective of justifying our assertion that the scatter increases as we go into the past.

The paper is organised as follows. Section 2 gives a brief review of the alternative model to be used and then develops the stochastic approach in 
it. Section 3 gives the new technique of finding the PDF for $h$ at any time in the past or present epochs and the results obtained on comparison between the theoretical and observational PDF's. The conclusions derived from it are discussed in section 4 .

\section{Stochastic approach in the new model}

In all FRW models, the Einstein equations, when combined with the conservation of total energy-momentum tensor can be written in terms of the Hubble parameter as

$$
\dot{H}=-H^{2}-\frac{4 \pi G}{3}(\rho+3 p)
$$

Overdot denotes time derivative. If we restrict ourselves to flat models, then (with $p=w \rho$ ),

$$
\dot{H}=-\frac{3}{2} H^{2}(1+w)
$$

In [2], we considered this flat case and assumed that $w$ is a Gaussian $\delta$-correlated Langevin force term with mean value zero. This means that the mean total pressure of the universe is zero, the same as that for dust. But many recent observations are incompatible with this model and hence, as mentioned in the introduction, we look for a more observationally correct, but simple model to apply our stochastic treatment.

The deterministic model [9] we propose to use is the one in which the total energy density obeys the condition $\rho+3 p=0$ and hence having a coasting 
$(a \propto t)$ evolution. This model is derived on the basis of some dimensional considerations in line with quantum cosmology. If we assume that the energy components in this model are dust and vacuum, then the above condition gives $\rho_{m} / \rho_{v}=2$ and if they are only radiation and vacuum, then $\rho_{r} / \rho_{v}=$ 1. In [9], it was shown that in this model, most outstanding cosmological problems like those of flatness, horizon, monopole, entropy, size and age of the universe, and the cosmological constant are absent. It was also shown that this model can solve the problem of generation of density perturbations at scales well above the present Hubble radius and that it can generate such density perturbations even after the era of nucleosynthesis. Though it is mentioned in the paper that recent observations on the redshift-apparent magnitude relation are at variance with the predictions of this model, in a recent communication [10], this issue was studied in detail and found that those observations do not provide any strong evidence against it.

It may be noted that the underlying model described above deviates from 'main stream' cosmology to some extent and the most significant cosmological observations such as nucleosynthesis, large scale structures and microwave background radiation, in this model are not well-studied. However, to repeat our statements above, we choose this model to implement our stochastic approach mainly due to its simplicity and its capability to explain the recent supernova data.

In view of the fact that this model has $w=-1 / 3$ in the deterministic 
case, we rewrite the above equation (4) with $w^{\prime} \equiv w+(1 / 3)$, as

$$
\dot{H}=-\frac{3}{2} H^{2}\left(\frac{2}{3}+w^{\prime}\right)
$$

We now assume that $w^{\prime}$ fluctuates about its zero mean value and is $\delta$ correlated. Making a substitution

$$
x \equiv \frac{1}{H}
$$

the above equation becomes

$$
\dot{x}=1+\frac{3}{2} w^{\prime}
$$

When $w^{\prime}=0$, this equation is analogous to that of a particle moving in a medium with constant velocity. With a fluctuating $w \prime$, the analogous particle is subjected to random forces as it moves. We write the FP equation for the distribution function for the variable $x$ as [7]

$$
\frac{\partial W^{\prime}}{\partial t}(x, t)=\left[-D^{(1)} \frac{\partial}{\partial x}+D^{(2)} \frac{\partial^{2}}{\partial x^{2}}\right] W^{\prime}(x, t),
$$

the solution of which gives the PDF $W^{\prime}$ for the variable $x$ at time $t$. Here the drift coefficient $D^{(1)}$ is the constant velocity term, equal to unity, and the diffusion coefficient $D^{(2)} \equiv D$ is assumed to have some constant value, to be determined from observation. The FP equation is solved by first assuming that the variables are separable;

$$
W^{\prime}(x, t)=\phi_{n}(x) e^{-\lambda_{n} t},
$$


where $\phi_{n}(x)$ and $\lambda_{n}$ are the eigen functions and eigen values of the FokkerPlanck operator

$$
L_{F P}=\left[-\frac{\partial D^{(1)}}{\partial x}(x)+\frac{\partial^{2} D^{(2)}}{\partial x^{2}}(x)\right] .
$$

Now we define two more functions in order to get a solution for the FP equation;

$$
\Phi(x) \equiv-\int \frac{D^{(1)}}{D^{(2)}} d x^{\prime}=-\frac{x}{D}
$$

and

$$
\psi_{n}(x)=\exp \left(\frac{\Phi}{2}\right) \phi_{n}(x)=\exp \left(-\frac{x}{2 D}\right) \phi_{n}(x)
$$

where $\Phi(x)$ is treated as a stochastic potential and $\psi_{n}(x)$ are the eigen functions of the Hermitian operator $L$ defined as

$$
L=\exp \left(\frac{\Phi}{2}\right) L_{F P} \exp \left(-\frac{\Phi}{2}\right)
$$

Making use of equations (8) and (11), the time independent part of FP equation becomes

$$
\frac{\partial^{2} \psi_{n}}{\partial x^{2}}(x)=\left[\frac{1}{4 D^{2}}-\frac{\lambda_{n}}{D}\right] \psi_{n}(x)=-k^{2} \psi_{n}(x) .
$$

Here,

$$
k= \pm\left[\frac{\lambda_{n}}{D}-\frac{1}{4 D^{2}}\right]^{\frac{1}{2}} .
$$

The most general solution to $(7)$ is

$$
W^{\prime}(x, t)=\sum_{n=0}^{\infty} c_{n} e^{-\lambda_{n} t} \phi_{n}(x)
$$


For $\lambda_{n}<\frac{1}{4 D}$, the solution $\psi_{n}(x)$ is exponentially diverging, which is not an admissible solution. Thus we conclude that $\lambda_{n} \geq \frac{1}{4 D}$ so that

$$
\phi_{k}(x)=A \exp \left(\frac{x}{2 D}+i k x\right)
$$

We make use of the completeness relation

$$
\delta\left(x-x^{\prime}\right)=\int_{-\infty}^{+\infty} \psi_{k}^{*}(x) \psi_{k}\left(x^{\prime}\right) d k
$$

to specify the initial condition $x=x^{\prime}$ at $t=t^{\prime}$. The transition probability for the variable to change from $x^{\prime}$ at time $t^{\prime}$ to $x$ at time $t$ is [7]

$$
\begin{aligned}
P\left(x, t \mid x^{\prime}, t^{\prime}\right) & =e^{L_{F P}\left(t-t^{\prime}\right)} \delta\left(x-x^{\prime}\right) \\
& =\exp \left[\frac{\Phi\left(x^{\prime}\right)}{2}-\frac{\Phi(x)}{2}\right] \int_{-\infty}^{+\infty} d k \psi_{k}^{*}(x) \psi_{k}\left(x^{\prime}\right) \exp \left[-\lambda(k)\left(t-t^{\prime}\right)\right] \\
& =\frac{1}{2 \sqrt{\pi D\left(t-t^{\prime}\right)}} \exp \left[-\frac{\left[\left(x-x^{\prime}\right)-\left(t-t^{\prime}\right)\right]^{2}}{4 D\left(t-t^{\prime}\right)}\right],
\end{aligned}
$$

where we have chosen $A=\frac{1}{\sqrt{2 \pi}}$ for normalisation purpose. For the special initial condition $W^{\prime}\left(x, t^{\prime}\right)=\delta\left(x-x^{\prime}\right)$, the transition probability $P\left(x, t \mid x^{\prime}, t^{\prime}\right)$ is the distribution function $W^{\prime}(x, t)$ [7]. In our case, we have the initial condition $x=x^{\prime}=0$ at $t=t^{\prime}=0$, so that

$$
W^{\prime}(x, t)=P(x, t \mid 0,0)=\frac{1}{2 \sqrt{\pi D t}} \exp \left[-\frac{(x-t)^{2}}{4 D t}\right]
$$

which is in Gaussian form and has its peak moving along in such a way that the expectation value of the variable is $\langle x\rangle=t$. This corresponds to the deterministic solution of (6). The width of the Gaussian is found from the 
variance $\sigma^{2}=\left\langle(x-\langle x\rangle)^{2}\right\rangle=2 D t$ and we find $\sigma \geq\langle x\rangle$ till $t=2 D$. We can immediately rewrite this distribution function in terms of the stochastic Hubble parameter $H$ as

$$
W^{\prime \prime}(H, t)=\frac{1}{2 H^{2}} \frac{1}{\sqrt{\pi D t}} \exp \left[-\frac{(1-H t)^{2}}{4 H^{2} D t}\right] .
$$

With $H=100 h \mathrm{~km} \mathrm{~s}^{-1} \mathrm{Mpc}^{-1}, t=t_{17} \times 10^{17} \mathrm{~s}$ and $D=D_{17} \times 10^{17} \mathrm{~s}$, the PDF $W(h, t)$ can be written as

$$
W(h, t)=\frac{3.0856}{2 h^{2}} \frac{1}{\sqrt{\pi D_{17} t_{17}}} \exp \left[-\frac{\left(3.0856-h t_{17}\right)^{2}}{4 h^{2} D_{17} t_{17}}\right] .
$$

For the range of values of interest, $1<t_{17}<5$ and $D_{17} \sim 10^{-3}, W(h, t)$ is

approximately a Gaussian. For fixed $D$, the half width of the Gaussian is found to increase as we go to lower values of $t$.

\section{PDF for $H$ from observational data}

From the Hubble diagram, one can find the PDF for the present Hubble constant $H_{0}$ in the following way. The traditional measure of distance to a $\mathrm{SN}$ is its observed distance modulus $\mu_{o}=m_{b o l}-M_{b o l}$, the difference between its bolometric apparent magnitude and absolute magnitude. In the FRW cosmology, the distance modulus is predicted from the source's redshift, $z$, according to

$$
\mu_{p}=5 \log \left[\frac{D_{L}}{1 \mathrm{Mpc}}\right]+25,
$$

where $D_{L}$ is the luminosity distance, found as

$$
D_{L}=r_{j} a\left(t_{0}\right)(1+z) \text {. }
$$


$a\left(t_{0}\right)$ is the present scale factor and $r_{j}$ is the radial coordinate of the SN Ia which emitted the light at some time $t_{j}$ in the past. In flat FRW models, $r_{j}$ is found as

$$
r_{j}=\int_{t_{j}}^{t_{0}} \frac{c d t}{a(t)}
$$

For the coasting model discussed in the previous section, for $k=0, r_{j}$ can be evaluated as

$$
r_{j}=\frac{c t_{0}}{a\left(t_{0}\right)} \int_{t_{j}}^{t_{0}} \frac{d t}{t}=\frac{c t_{0}}{a\left(t_{0}\right)} \ln (1+z)
$$

so that

$$
D_{L}=\frac{c(1+z)}{H_{0}} \ln (1+z) .
$$

One can use this expression in (22) to obtain the predicted distance modulus of an object with redshift $z$. Conventionally, assuming that the observed and predicted distance moduli coincide, one can find a value of $H_{0}$. For a collection of objects, also one can find the likelihood for $H_{0}$, from a $\chi^{2}$ statistic;

$$
\chi^{2}=\sum_{i} \frac{\left(\mu_{p, i}-\mu_{o, i}\right)^{2}}{\sigma_{i}^{2}},
$$

where $\sigma_{i}$ is the total uncertainty in the corrected peak magnitude of SN Ia. For the special model we are considering, $h$ is the only parameter and the normalised PDF can now be obtained as [3]

$$
p\left(h \mid \mu_{0}\right)=\frac{\exp \left(-\chi^{2} / 2\right)}{\int_{-\infty}^{\infty} d h \exp -\chi^{2} / 2} .
$$

As in [2], we compute $p\left(h \mid \mu_{o}\right)$ for the new model using the SNe data in [1], which corresponds to their Fit $\mathrm{C}$ and attempt to compare $p\left(h \mid \mu_{o}\right)$ with 
the $\mathrm{PDF} W\left(h, t_{0}\right)$ to evaluate the diffusion constant $D$ appearing in this expression. It is found that the two curves, shown in figure 1, coincide for a value of $D \approx 2.36 \times 10^{13} \mathrm{~s}$. (This corresponds to an age $4.8583 \times 10^{17} \mathrm{~s}$.) Since our primary objective is to make an order of magnitude evaluation of $D$, we chose a fiducial absolute magnitude for SN Ia in computing $\mu_{o}$, equal to $-19.3 \mathrm{mag}$. Slight variations in this quantity will not significantly affect $D$, though the best fit value for $h$ may change.

In the above, we compared the theoretical and observational PDF's for the same alternative model and thus it does not have the first shortcoming mentioned in the introduction. The other incompatibility which still exists can be explicitly stated as follows: $W\left(h, t_{0}\right)$ is the PDF for the Hubble parameter of the present universe and it contains the diffusion constant $D$. But $p\left(h \mid \mu_{o}\right)$, which we attempt to identify with $W\left(h, t_{0}\right)$, depends on the scatter in the Hubble diagram for all ranges of redshift. For instance, if we include more high redshift objects in our sample, the scatter would be larger and hence the half-width of the distribution $p\left(h \mid \mu_{o}\right)$ will be larger. This, in turn, will affect the computed value of $D$, which is quite unreasonable.

This problem can, however, be overcome if we agree to compute $p\left(h_{j} \mid \mu_{o j}\right)$ for each value of redshift $z$ (or for small enough redshift intervals centred about such values), and compare these with $W\left(h_{j}, t_{j}\right)$ that corresponds to the same epoch $t_{j}$. To do this, we modify (26) by re-evaluating $r_{j}$ in (25) in 
a different way. One can also write, for the new deterministic model

$$
r_{j}=\frac{c t_{j}}{a\left(t_{j}\right)} \int_{t_{j}}^{t_{0}} \frac{d t}{t}=\frac{c}{H_{j} a\left(t_{j}\right)} \ln (1+z),
$$

so that

$$
D_{L}=\frac{c(1+z)^{2}}{H_{j}} \ln (1+z)
$$

Evaluating $\mu_{p}$ using this expression in (22), we can calculate $\chi^{2}$ and hence also $p\left(h_{j} \mid \mu_{o j}\right)$, which is the PDF for the Hubble parameter at some particular value of $z$. We divide the data in [1] for various ranges around $z=0.05,0.15$, $0.35,0.45,0.55$ and 0.65 , each with $\Delta z=0.05$. The PDF for the average Hubble parameter for such intervals is calculated with an expression identical to (28). The results are plotted in figure 2 along with the corresponding $W\left(h_{j}, t_{j}\right)$ which overlaps with them. The relevant parameters are given in table 1.

\section{Conclusions}

It can be noted from figure (2) and table 1 that for the intervals with larger values of $z$, the $68.3 \%$ credible region of $p\left(h \mid \mu_{o j}\right)$ has a half-width $\sigma_{h}$ which also increases (The intervals with centre at $z=0.15$ and 0.35 are exceptions to it, but this may be due to the fact that these intervals contain only very few objects. As more SN Ia are observed in these redshift bins, an accurate picture will emerge.). This behaviour is the one expected from theory, as noted

while plotting the theoretical PDF (21). The value of the diffusion constant 
$D$ evaluated for various intervals, however, does not show any dependence on $z$. This justifies our assumption (taken for the sake of simplicity) that $D$ is some constant.

As remarked in the introduction, a novel feature of the present analysis is that we compute the PDF for $H$ at various epochs in the past. However, there is a limitation, even in the present analysis; the intervals we consider are with $\Delta z=0.05$ and this value may not be small enough to give correct answers. The procedure can be improved very much in the future, when the number of observed SN Ia becomes large.

It should be kept in mind that the measurements are made with finite accuracy and hence have a scatter arising from intinsic statistics of error. Here we have not shown that the scatter in the Hubble diagram is significantly more than that expected from the known errors. The present theory can survive only if $p\left(h \mid \mu_{o}\right)$ does not become narrower with increase in accuracy of measurement. Until it is confirmed by future observations like the Supernova Acceleration Probe (SNAP) [11], which aims to give precise luminosity distance of $\approx 2000 \mathrm{SN}$ Ia upto $z=1.7$ every year, the computed value of the diffusion constant can not be claimed as accurate. Given this circumstance, it is safer to conclude for the present that the scatter in the SN Ia data places limits on the stochastic variation in the expansion rate and consequently, on the stochastic variation of the equation of state. In other words, it is justifiable to consider the value of $D$ computed by us using SN 
Ia data as an upper limit to a possible diffusion constant for the universe.

\section{Acknowledgment}

We are thankful to Professor Jayant Narlikar for valuable discussion and to the referee for his suggestions. CS thanks CSIR, New Delhi for the award of a Research Fellowship.

\section{References}

[1] S Perlmutter et al, Astrophys. J. 517, 565 (1999)

[2] C Sivakumar, M V John and K Babu Joseph, Pramana-J. Phys. 56, 477 (2001); C Sivakumar, M V John and K Babu Joseph, Submitted (2000)

[3] A G Riess et al, Astron. J. 116, 1009 (1998)

[4] W L Freedman et al, Nature 371, 757 (1994) M J Pierce, D L Welch, R D McClure, S van den Bergh, R Racine, and P B Stetson, Nature 371, 385 (1994) I Zehavi and A Dekel, Nature 401, 252 (1999) L M Krauss, Astrophys. J. 501, 461 (1998)

[5] M Kamionkowski and N Toumbas, Phys. Rev. Lett. 77, 587 (1996)

[6] R R Caldwell, R Dave and P J Steinhardt, Phys. Rev. Lett. 80, 1582 (1998) I Zlatev, L Wang and P J Steinhardt, Phys. Rev. lett. 82, 896 (1999) 
[7] H Risken, The Fokker-Planck Equation (Springer-Verlag, New York, 1984)

[8] T Padmanabhan, To appear in the proceedings of XIII Rencontres de Blois-Frontiers of the universe, June 17-23 (2001), gr-qc/0112068

[9] M V John and K Babu Joseph, Phys. Rev. D61, 087304 (2000)

[10] M V John and J V Narlikar, Phys. Rev. D65, 043506 (2002)

[11] J Weller and A Albrecht, astro-ph/0106079 
Table 1

\begin{tabular}{l|l|l|l|l|l}
\hline $\begin{array}{l}\text { Redshift } \\
\mathrm{z}\end{array}$ & $\begin{array}{l}\text { No. of SNe } \\
\text { in the } \\
\text { interval } z \pm 0.05\end{array}$ & $\begin{array}{l}\text { Best fit } \\
\text { value of } \\
\mathrm{h}\end{array}$ & $\begin{array}{l}\text { Standard } \\
\text { deviation } \\
\sigma_{h}\end{array}$ & $\begin{array}{l}\text { Age in } \\
\text { units of } 10^{17} \mathrm{~s} \\
t_{17}\end{array}$ & $\begin{array}{l}\text { Diffusion } \\
\text { constant } \\
\mathrm{D} \mathrm{s}\end{array}$ \\
\hline 0.05 & 15 & 0.693 & 0.011 & 4.4502 & $0.5775 \times 10^{14}$ \\
0.15 & 3 & 0.772 & 0.025 & 3.9987 & $2.147 \times 10^{14}$ \\
0.35 & 5 & 0.830 & 0.024 & 3.7204 & $1.5 \times 10^{14}$ \\
0.45 & 15 & 0.875 & 0.017 & 3.528 & $1.655 \times 10^{14}$ \\
0.55 & 7 & 0.985 & 0.026 & 3.1345 & $1.12 \times 10^{14}$ \\
0.65 & 6 & 1.043 & 0.030 & 2.959 & $1.226 \times 10^{14}$ \\
\hline
\end{tabular}


Figure caption:

Figure 1: Observational and theoretical PDF's vs $h$, using the apparent magnitude-redshift data for Type Ia supernovae as given in [1], which corresponds to their Fit $\mathrm{C}$. The continuous line is for the observational PDF whereas the dotted line gives the theoretical PDF.

Figure 2: Observational and theoretical PDF's vs $h_{j}$ (where $h_{j}$ corresponds to the Hubble parameter for the epoch centered about redshifts $z=0.05,0.15,0.35,0.45,0.55,0.65)$, using the apparent magnitude-redshift data for Type Ia supernovae as given in [1], which corresponds to their Fit $\mathrm{C}$ and which lies in the interval $z \pm 0.05$. 